\title{
STOCHASTIC BACKPRESSURE IN ENERGY HARVESTING NETWORKS
}

\author{
Miguel Calvo-Fullana ${ }^{\dagger}$, Javier Matamoros $^{\dagger}$, Carles Antón-Haro $^{\dagger}$ and Alejandro Ribeiro \\ ${ }^{\dagger}$ Centre Tecnològic de Telecomunicacions de Catalunya (CTTC), Barcelona, Spain \\ ${ }_{\ddagger}^{\ddagger}$ University of Pennsylvania, Philadelphia, USA
}

\begin{abstract}
In this paper, we study the problem of jointly routing and scheduling traffic in an energy harvesting network. To this end, we leverage stochastic dual descent methods to propose a generalization of the well-known backpressure algorithm to energy harvesting networks. We name this policy energy harvesting backpressure (EH-BP) and show that it satisfies the fundamental property of backpressure-type algorithms. Namely, if given data arrival rates can be supported by given energy arrival rates and some routing-scheduling policy, they can be supported by the EH-BP policy. Numerical results attest to the properties of the proposed policy.
\end{abstract}

Index Terms-Energy harvesting, wireless networks, backpressure, routing, scheduling.

\section{INTRODUCTION}

Recently, Energy Harvesting (EH) has emerged as a technology capable of allowing network nodes to replenish their batteries using environmental energy sources—such as, solar radiation, radio waves or vibration [1]. This, in turn, potentially allows the network nodes to operate for an infinite lifetime. However, the intermittent and random nature of the energy supply makes it necessary to take a new approach to the design of communication policies. This has led to a great deal of research interest in $\mathrm{EH}$-powered communication, with problems ranging from throughput maximization [2-5], sourcechannel coding [6-8], estimation [9-11] and others being studied (see [12] for a comprehensive overview).

A problem that often arises in wireless networks is the need to route information through the neighboring nodes. In this regard, the design of both routing and scheduling algorithms for energy harvesting networks has been previously considered in the literature. For example, the authors in [13] propose an EH-aware routing scheme that is asymptotically optimal with respect to the network size. In [14], the authors adress the EH scheduling problem for both single-hop and multi-hop networks, and provide a joint admission control and routing policy. In the same line, the authors in [15] propose a policy which improves on the multi-hop performance bounds of [14].

In this paper, we study the problem of jointly routing and scheduling data packets in an energy harvesting network. Each node independently generates traffic for delivery to a specific destination and collaborates with the other nodes in the network to ensure the delivery of all data packets. In this way, each node decides the next suitable hop of for each packet in its queue (routing), and when to transmit it (scheduling). The solution to this problem-when

This work was partially supported by the Catalan government under grant SGR2014-1567; the Spanish government under grant TEC2013-44591P (INTENSYV), and grant PCIN-2013-027 (E-CROPS) in the framework of the ERA-NET CHIST-ERA program.

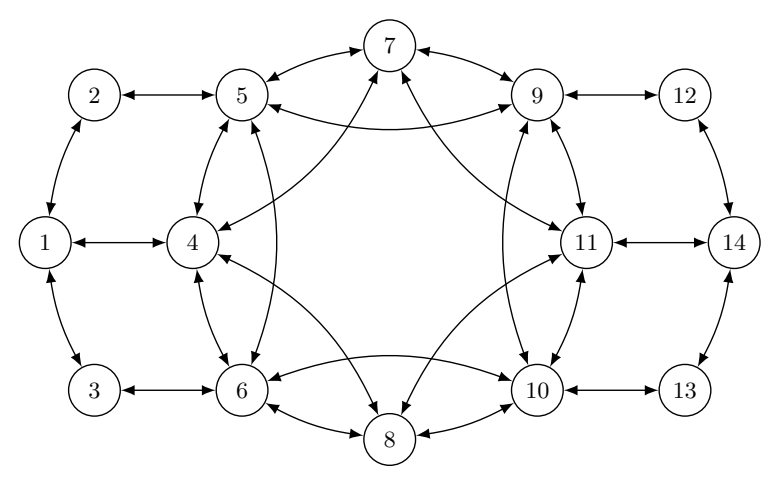

Fig. 1. Example of a communications network.

the nodes are not EH-powered-is given by the backpressure (BP) algorithm [16].

The works [14] and [15] considered a similar problem which consists in finding admission control and resource allocation policies that satisfy network stability and energy causality while attaining close to optimal network utility. In this work, instead, our goal is to find stabilizing policies given the data rates. Also, while previous works $[14,15]$ require the data and energy arrival process to be i.i.d. or Markov, we only require them to be ergodic, which is a weaker requirement. Furthermore, our approach to the problem is also markedly different. While the works [14] and [15] relied on queueing theory and Lyapunov drift arguments to find stabilizing policies, we instead interpret the scheduling and routing problem as a stochastic optimization problem. This allows us to resort to a modified dual stochastic subgradient descent algorithm $[17,18]$ to solve the joint routing-scheduling problem. In the proposed algorithm, which we denote by energy harvesting backpressure (EH-BP), the nodes track the pressure of the information flows by computing the difference between the Lagrange multipliers associated to their queue stability constraints and the ones of their neighbors (instead of their data queues as in the classical BP algorithm). Then, the Lagrange multipliers associated with the energy harvesting process reduce the pressure when the stored energy in the node decreases. Hence, we identify a direct relationship between the values of the dual variables and the battery and data queues they represent. Finally, we asses the performance of the EH-BP algorithm by means of simulations.

\section{SYSTEM MODEL}

Consider a communication network given by the graph $\mathcal{G}=(\mathcal{N}, \mathcal{E})$, where $\mathcal{N}$ is the set of $N$ nodes in the network and $\mathcal{E} \subseteq \mathcal{N} \times \mathcal{N}$ is the set of communication links, such that if node $i$ is capable of communicating with node $j$, we have $(i, j) \in \mathcal{E}$. Moreover, we 
define the neighborhood of node $i$ as the set $\mathcal{N}_{i}=\{j \mid(i, j) \in \mathcal{E}\}$. The network supports $K$ information flows (which we index by the set $\mathcal{K}$ ), where for a flow $k \in \mathcal{K}$, the destination node is denoted by $N_{(\text {dest })}^{k}$. At a time slot $t$, each $k \in \mathcal{K}$ flow at the $i$-th node generates $a_{i}^{k}[t]$ packets to be delivered to the node $N_{(\text {dest })}^{k}$. This packet arrival process is assumed to be stationary with mean $\mathbb{E}\left[a_{i}^{k}[t]\right]=a_{i}^{k}$. At the same time, the $i$-th node routes $r_{i j}^{k}[t]$ packets to its neighbors $j \in$ $\mathcal{N}_{i}$, while simultaneously being routed $r_{j i}^{k}[t]$ packets. For simplicity, at each time slot, we restrict each node to route one single packet to its neighbors. Therefore, the nodes have the following routing constraint

$$
\sum_{k \in \mathcal{K}} \sum_{j \in \mathcal{N}_{i}} r_{i j}^{k}[t] \leq 1, \quad i \in \mathcal{N}
$$

Furthermore, each node in the network keeps track of the number of packets awaiting to be transmitted for each flow. Denoting by $q_{i}^{k}[t]$ the $k$-th flow data queue at the $i$-th node and time slot $t$, the evolution of the queue is given by

$$
q_{i}^{k}[t+1]=q_{i}^{k}[t]+a_{i}^{k}[t]+\sum_{j \in \mathcal{N}_{i}} r_{j i}^{k}[t]-\sum_{j \in \mathcal{N}_{i}} r_{i j}^{k}[t]
$$

for all $i \in \mathcal{N}$ and $k \in \mathcal{K}$. We consider that the network nodes are powered by energy harvesting. At time slot $t$, the $i$-th node harvests $e_{i}[t]$ units of energy, where the energy harvesting process is assumed to be stationary with mean $\mathbb{E}\left[e_{i}[t]\right]=e_{i}$. We consider a normalized energy harvesting process, where the routing of one packet consumes one unit of energy. Furthermore, we consider packet transmission to be the only energy-consuming action taken by the nodes. Under these conditions and denoting by $b_{i}[t]$ the energy stored in the $i$-th node's battery at time $t$, the following energy causality constraint must be satisfied for all time slots

$$
\sum_{k \in \mathcal{K}} \sum_{j \in \mathcal{N}_{i}} r_{i j}^{k}[t] \leq b_{i}[t], \quad i \in \mathcal{N}
$$

Additionally, we consider that nodes have a finite battery of capacity $b_{i}^{\max }$. Then, we can write the battery dynamics as

$$
b_{i}[t+1]=\left[b_{i}[t]-\sum_{k \in \mathcal{K}} \sum_{j \in \mathcal{N}_{i}} r_{i j}^{k}[t]+e_{i}[t]\right]_{0}^{b_{i}^{\max }}, \quad i \in \mathcal{N} .
$$

where $[\cdot]_{0}^{b_{i}^{\max }}$ denotes the projection to the interval $\left[0, b_{i}^{\max }\right]$. Our goal is to determine routing policies $r_{i j}^{k}[t]$ such that the queues (2) remain stable while satisfying the routing (1) and energy causality (3) constraints. By grouping the all the queues in a vector $\mathbf{q}[t]=$ $\left\{q_{i}[t]\right\}$, we say that the routing policies $r_{i j}^{k}[t]$ guarantee stability if there exists $Q$ such that for some arbitrary time $T$ we have

$$
\operatorname{Pr}\left\{\max _{t \geq T}\|\mathbf{q}[t]\| \leq Q \mid \mathbf{q}(T)\right\}=1
$$

This is to say that, almost surely, no queue becomes arbitrarily large. In turn, we can guarantee this if the average rate at which packets enter the queues is lower than the rate at which they exit them. In order to formulate this problem, let us denote the ergodic limits of processes $a_{i}^{k}[t], r_{i j}^{k}[t]$ and $e_{i}[t]$ by $a_{i}^{k}, r_{i j}^{k}$, and $e_{i}$, respectively. Then, by defining the routing weights $w_{i}^{k}$, we can pose the following net- work throughput maximization problem

$$
\begin{array}{ll}
\underset{\sum_{k, j} r_{i j}^{k} \leq 1}{\operatorname{maximize}} & \sum_{i \in \mathcal{N}} \sum_{k \in \mathcal{K}} \sum_{j \in \mathcal{N}_{i}} w_{i}^{k} r_{i j}^{k} \\
\text { subject to } & a_{i}^{k} \leq \sum_{j \in \mathcal{N}_{i}} r_{i j}^{k}-\sum_{j \in \mathcal{N}_{i}} r_{j i}^{k}, \quad k \in \mathcal{K}, i \in \mathcal{N} \\
& \sum_{k \in \mathcal{K}} \sum_{j \in \mathcal{N}_{i}} r_{i j}^{k} \leq e_{i}, \quad i \in \mathcal{N}
\end{array}
$$

where we have left the routing constraints (1) implicit, and the optimization is over the nonnegative routing variables $r_{i j}^{k} \geq 0$. Furthermore, we have substituted the per time slot constraints (1) and (3) for average ones. If there exists routing variables $r_{i j}^{k}$ satisfying constraint (6b), then the queue evolution (2) follows a supermartingale, and the stability condition (5) is then guaranteed by the martingale convergence theorem [19]. Then, assuming data and energy arrival rates satisfying (6b) and (6c) exist, we will design an algorithm such that the instantaneous routing variables $r_{i j}^{k}[t]$ satisfy $\mathbb{E}\left[r_{i j}^{k}[t]\right]=r_{i j}^{k}$ and the constraints (1) and (3) are satisfied for all time slots.

\section{STOCHASTIC BACKPRESSURE ALGORITHM}

Let us define the vector $\mathbf{r}=\left\{r_{i j}^{k}\right\}$ collecting the routing variables and the vector $\boldsymbol{\lambda}=\left\{\gamma_{i}^{k}, \beta_{i}\right\}$ collecting all the queue multipliers $\gamma_{i}^{k}$ and battery multipliers $\beta_{i}$. Then, we can write the Lagrangian of the optimization problem (6) as follows

$$
\begin{aligned}
\mathcal{L}(\mathbf{r}, \boldsymbol{\lambda}) & =\sum_{i \in \mathcal{N}} \sum_{k \in \mathcal{K}} \sum_{j \in \mathcal{N}_{i}} w_{i}^{k} r_{i j}^{k} \\
& +\sum_{k \in \mathcal{K}} \sum_{i \in \mathcal{N}} \gamma_{i}^{k}\left(\sum_{j \in \mathcal{N}_{i}} r_{i j}^{k}-\sum_{j \in \mathcal{N}_{i}} r_{j i}^{k}-a_{i}^{k}\right) \\
& +\sum_{i \in \mathcal{N}} \beta_{i}\left(e_{i}-\sum_{k \in \mathcal{K}} \sum_{j \in \mathcal{N}_{i}} r_{i j}^{k}\right) .
\end{aligned}
$$

The Lagrange dual function is then given by

$$
g(\boldsymbol{\lambda})=\max _{\mathbf{r} \geq 0} \mathcal{L}(\mathbf{r}, \boldsymbol{\lambda}),
$$

and we can reorder the Lagrangian (7) to allow for a separate maximization over network nodes, where each node only needs the queue multipliers of its neighboring nodes. The routing variables can then be obtained as follows

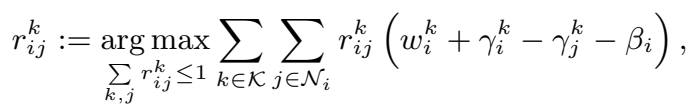

for $i \in \mathcal{N}$. An immediate problem that arises when trying to solve this problem is that network nodes have no knowledge of the data arrival rates $a_{i}^{k}$ nor the energy harvesting rates $e_{i}$. Nonetheless, the nodes observe the instantaneous rates $a_{i}^{k}[t]$ and $e_{i}[t]$, hence we can resort to using these instantaneous variables. To solve (9) it suffices to find the flow over the neighboring nodes with the largest differential $w_{i}^{k}+\gamma_{i}^{k}[t]-\gamma_{j}^{k}[t]-\beta_{i}[t]$ and if it is positive, set its routing variable $r_{i j}^{k}[t]$ to one while the other variables are kept to zero.

Now, since the dual function (8) is convex, we can perform a stochastic subgradient descent by defining the following dual updates

$$
\gamma_{i}^{k}[t+1]:=\left[\gamma_{i}^{k}[t]+a_{i}^{k}[t]+\sum_{j \in \mathcal{N}_{i}} r_{j i}^{k}[t]-\sum_{j \in \mathcal{N}_{i}} r_{i j}^{k}[t]\right]_{0}^{\gamma_{i}^{k, \max }}
$$




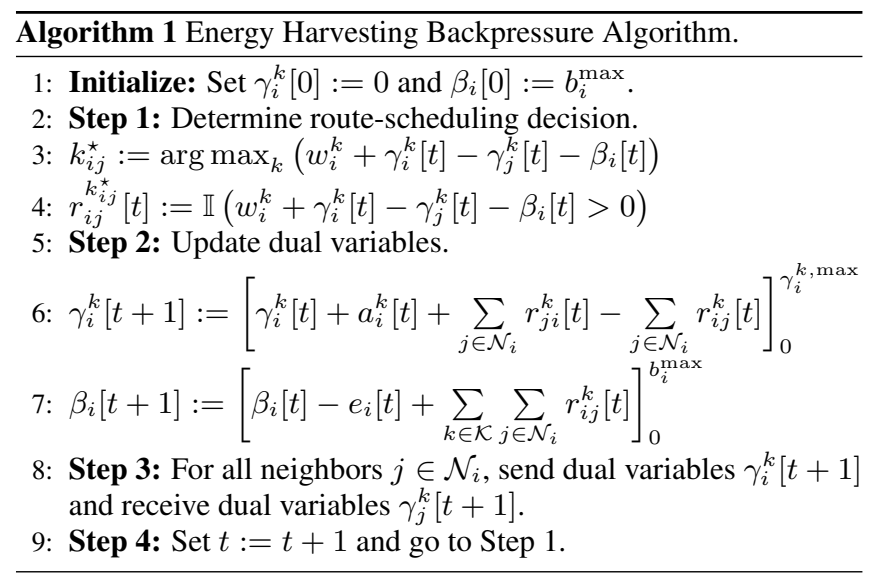

$$
\beta_{i}[t+1]:=\left[\beta_{i}[t]-e_{i}[t]+\sum_{k \in \mathcal{K}} \sum_{j \in \mathcal{N}_{i}} r_{i j}^{k}[t]\right]_{0}^{b_{i}^{\max }}
$$

where we have used a unit step size and projected the multipliers to a restricted interval. Denote by $\gamma_{i}^{k, \star}$ and $\beta_{i}^{\star}$ the optimal Lagrange multipliers associated with constraint $(6 \mathrm{~b})$ and $(6 \mathrm{c})$, respectively. Then if $\gamma_{i}^{k, \star} \in\left[0, \gamma_{i}^{k, \max }\right]$ and $\beta_{i}^{\star} \in\left[0, \beta_{i}^{\max }\right]$ the ergodic limit convergence of the dual updates (10) and (11) to these optimal values can be ensured. For compactness, we collect the dual updates in the vector $\boldsymbol{\lambda}[t+1]:=[\boldsymbol{\lambda}[t]-\mathbf{s}[t]]_{0}^{\boldsymbol{\lambda}_{\max }}$, where $\mathbf{s}[t]$ corresponds to the vector collecting the stochastic subgradients and $\lambda^{\max }$ the thresholds $\left\{\gamma_{i}^{k, \max }, \beta_{i}^{\max }\right\}$. Then, the convergence condition is given by $\boldsymbol{\lambda}^{\star} \in\left[0, \boldsymbol{\lambda}^{\max }\right]$, where $\boldsymbol{\lambda}^{\star}$ is the vector collecting all the optimal Lagrange multipliers.

With these definitions in place, we can draw a comparison between the dual updates and the original queue and battery dynamics. First, notice that the the dual update (10) acts as a thresholded version of the data queue (2). And, in a similar way, the dual update (11) mirrors the battery dynamics (4), as they can be written as $b_{i}[t]=b_{i}^{\max }-\beta_{i}[t]$. Then, an appropriate choice of battery capacity can be made in order to satisfy the energy causality constraints (3).

Proposition 1 (Energy Causality). Let the battery capacity satisfy $b_{i}^{\max } \geq w_{i}^{k}+\gamma_{i}^{k, \max }$, for all $i, k$, then Algorithm 1 satisfies the energy consumption causality constraint (3) for all time slots.

Proof. In order to satisfy (3), we must certify that no transmission occurs when there is no available energy in the battery. That is, $r_{i j}^{k}=0$ for all $j, k$ if $b_{i}[t]=0$. Then, it suffices the ensure that $w_{i}^{k}+\gamma_{i}^{k}[t]-\gamma_{j}^{k}[t]-\beta_{i}[t]<0$ for all $t$. When $b_{i}[t]=0$, the battery dual update takes the value $\beta_{i}[t]=b_{i}^{\max }$ and by the dual update (10), the difference $\gamma_{i}^{k}[t]-\gamma_{j}^{k}[t]$ is upper bounded by $\gamma_{i}^{k \text {,max }}$. We can write $w_{i}^{k}+\gamma_{i}^{k, \max }-b_{i}^{\max } \leq 0$, and since $b_{i}^{\max } \geq w_{i}^{k}+\gamma_{i}^{k, \max }$, this ensures that the maximization in (9) leads to $r_{i j}^{\bar{k}}=0$. Hence, ensuring no transmission occurs.

This proposition attests to the existence of a tradeoff between the weights $w_{i}^{k}$ and the battery requirements $b_{i}^{\max }$ of the node. A node with negative weight will have more packets being queued while, at the same time, requiring a smaller battery. On the contrary, a positive weight $w_{i}^{k}$ will lead to smaller queues while increasing the battery requirements. Setting the weight to zero leads to the classical backpressure algorithm, adapted to the energy harvesting scenario. In this case, by Proposition 1 the threshold of both multipliers can be chosen to be equal, i.e. $\gamma_{i}^{k, \max }=b_{i}^{\max }$.

\section{STABILITY ANALYSIS}

In this section, we establish the stability properties of the proposed EH-BP algorithm. In order to prove the queue stability of problem (6) when solved by Algorithm 1 we first need the following lemma

Lemma 2. Consider the dual updates of Algorithm 1 given by (10) and (11), and let $\mathbb{E}\left[\|\mathbf{s}[t]\|^{2} \mid \boldsymbol{\lambda}[t]\right] \leq S^{2}$ be a bound on the second moment of the norm of the stochastic subgradients $\mathbf{s}[t]$. Assume that the dual variable $\boldsymbol{\lambda}[T]$ is given for an arbitrary time $T$ and define as $\boldsymbol{\lambda}_{\text {best }}[t]:=\arg \min _{\boldsymbol{\lambda}[l]} g(\boldsymbol{\lambda}[l])$ the dual variable leading to the best value of the of the dual function for the interval $l \in[T, t]$. Then, if $\boldsymbol{\lambda}^{\star} \in\left[0, \boldsymbol{\lambda}^{\mathrm{max}}\right]$, we have

$$
\lim _{t \rightarrow \infty} g\left(\boldsymbol{\lambda}_{\text {best }}[t] \mid \boldsymbol{\lambda}[T]\right) \leq g\left(\boldsymbol{\lambda}^{\star}\right)+\frac{S^{2}}{2} \quad \text { a.s }
$$

Proof. Omitted due to space limitations.

This lemma states that with probability one the gap between the dual function and its optimal value closes to $S^{2} / 2$ at least once as $t$ increases. Moreover, since we can choose $T$ arbitrarily, we can conclude that this gap closes an infinite amount of times. We will use this lemma to prove the feasibility of Algorithm 1.

Proposition 3 (Feasibility). Assume there exist strictly feasible routing variables $r_{i j}^{k}$ such that $\sum_{j \in \mathcal{N}_{i}} r_{i j}^{k}-\sum_{j \in \mathcal{N}_{i}} r_{j i}^{k}-a_{i}^{k}>C$ and $e_{i}-\sum_{k \in \mathcal{K}} \sum_{j \in \mathcal{N}_{i}} r_{i j}^{k}>C$, for some $C>0$. Furthermore, assume the optimal dual variables satisfy $\boldsymbol{\lambda}^{\star} \in\left[0, \boldsymbol{\lambda}^{\max }\right]$ and let $\boldsymbol{\lambda}^{\max }>\left(g\left(\boldsymbol{\lambda}^{\star}\right)+S^{2} / 2-\sum_{i \in \mathcal{N}} \sum_{k \in \mathcal{K}} \sum_{j \in \mathcal{N}_{i}} w_{i}^{k} r_{i j}^{k}\right) / C$ element-wise. Then, the constraints (6b) and (6c) are almost surely satisfied by Algorithm 1.

Proof. First, let us collect the feasible routing variables in the vector $\mathbf{r}_{0}=\left\{r_{i j}^{k}\right\}$. Then, if there exist strictly feasible variables $r_{i j}^{k}$ we can bound the value of the dual function $g(\boldsymbol{\lambda})$ as follows. The dual function is defined as the maximum over primal variables $g(\boldsymbol{\lambda})=\max _{\mathbf{r} \geq 0} \mathcal{L}(\mathbf{r}, \boldsymbol{\lambda})$, hence $g(\boldsymbol{\lambda}) \geq \mathcal{L}\left(\mathbf{r}_{0}, \boldsymbol{\lambda}\right)$ and using the $\sum_{j \in \mathcal{N}_{i}} r_{i j}^{k}-\sum_{j \in \mathcal{N}_{i}} r_{j i}^{k}-a_{i}^{k}>C$ and $e_{i}-\sum_{k \in \mathcal{K}} \sum_{j \in \mathcal{N}_{i}} r_{i j}^{k}>C$ terms establish the following bound

$$
g(\boldsymbol{\lambda}) \geq \sum_{i \in \mathcal{N}} \sum_{k \in \mathcal{K}} \sum_{j \in \mathcal{N}_{i}} w_{i}^{k} r_{i j}^{k}+C \boldsymbol{\lambda}^{T} \mathbf{1} .
$$

Then, by reordering terms we obtain the following upper bound on the dual variables

$$
\boldsymbol{\lambda} \leq \frac{1}{C}\left(g(\boldsymbol{\lambda})-\sum_{i \in \mathcal{N}} \sum_{k \in \mathcal{K}} \sum_{j \in \mathcal{N}_{i}} w_{i}^{k} r_{i j}^{k}\right) .
$$

By Lemma 2 we can certify the existence of a time $t \geq T_{0}$ for which $g(\boldsymbol{\lambda}[t]) \leq g\left(\boldsymbol{\lambda}^{\star}\right)+S^{2} / 2$. Hence, we write

$$
\boldsymbol{\lambda}[t] \leq \frac{1}{C}\left(g\left(\boldsymbol{\lambda}^{\star}\right)+\frac{S^{2}}{2}-\sum_{i \in \mathcal{N}} \sum_{k \in \mathcal{K}} \sum_{j \in \mathcal{N}_{i}} w_{i}^{k} r_{i j}^{k}\right) \quad t \geq T_{0} .
$$

Now, recall that the feasibility conditions (6b) and (6c) are given by the ergodic limits

$$
\lim _{t \rightarrow \infty} \frac{1}{t} \sum_{l=1}^{t}\left(\sum_{j \in \mathcal{N}_{i}} r_{i j}^{k}[l]-\sum_{j \in \mathcal{N}_{i}} r_{j i}^{k}[l]-a_{i}^{k}[l]\right) \geq 0, \quad \text { a.s }
$$




$$
\lim _{t \rightarrow \infty} \frac{1}{t} \sum_{l=1}^{t}\left(e_{i}[l]-\sum_{k \in \mathcal{K}} \sum_{j \in \mathcal{N}_{i}} r_{i j}^{k}[l]\right) \geq 0, \quad \text { a.s }
$$

which, by recalling that the constraints are simply the stochastic subgradients of the problem, they can also be written in compact form as

$$
\lim _{t \rightarrow \infty} \frac{1}{t} \sum_{l=1}^{t} \mathbf{s}[l] \geq 0, \quad \text { a.s. }
$$

We will prove feasibility by contradiction. Start by assuming that equation (18) is unfeasible, so there exists a time $t \geq T_{1}$, for which there is a $\delta>0$ constant such that

$$
\frac{1}{t} \sum_{l=1}^{t} \mathbf{s}[l] \leq-\delta
$$

Furthermore, since the multiplier updates (10) and (11) are given by $\boldsymbol{\lambda}[t+1]:=[\boldsymbol{\lambda}[t]-\mathbf{s}[t]]_{0}^{\boldsymbol{\lambda}_{\max }}$, there is a time index $T_{1}$ such that for $t \geq T_{1}$ we have $\boldsymbol{\lambda}[t]=\boldsymbol{\lambda}_{\max }$. But we also have that $\boldsymbol{\lambda}^{\max }$ is lower bounded by

$$
\boldsymbol{\lambda}^{\max }>\frac{1}{C}\left(g\left(\boldsymbol{\lambda}^{\star}\right)+\frac{S^{2}}{2}-\sum_{i \in \mathcal{N}} \sum_{k \in \mathcal{K}} \sum_{j \in \mathcal{N}_{i}} w_{i}^{k} r_{i j}^{k}\right)
$$

which is a contradiction of (15). Thus, the feasibility conditions (16) and (17) are satisfied.

Remark 4. We note that the requirement on $\lambda^{\max }$ in the preceding proposition is admittedly very loose. However, in the numerical results section we will see that less conservative thresholds work appropriately. Nonetheless, we leave the derivation of tighter bounds for future work. sition.

Finally, queue stability follows directly from the previous propo-

Corollary 5 (Queue Stability). Consider the conditions of Proposition 3 , then the queues are stable in the sense of (5).

Proof. Denote by $\mathcal{F}_{i}^{k}[t]$ the sequence of nested $\sigma$-algebras measuring $q_{i}^{k}[l]$ for $l \in\{0, \ldots, t\}$. Then, since by Proposition 3 the ergodic limits generated by Algorithm 1 satisfy $\sum_{j \in \mathcal{N}_{i}} r_{i j}^{k}-\sum_{j \in \mathcal{N}_{i}} r_{j i}^{k}-$ $a_{i}^{k} \geq 0$, the queue evolution (2) obeys the supermartingale expression $\mathbb{E}\left[q_{i}^{k}[t+1] \mid \mathcal{F}_{i}^{k}[t]\right] \leq q_{i}^{k}[t]$. By the supermartingale convergence theorem [19, Theorem 5.2.9], the sequence $q_{i}^{k}[t]$ converges almost surely, therefore satisfying the stability condition (5).

\section{NUMERICAL RESULTS}

In this section we conduct numerical experiments to evaluate the performance of the proposed EH-BP algorithm. We consider the network shown in Figure 1, where the nodes 1 and 14 act as sink nodes and the rest of the nodes support a single flow with packet arrival rates of $a_{i}^{k}=0.4$. Moreover, we consider the nodes to be harvesting energy at a rate of $e_{i}=1$ and storing it in a battery of capacity $b_{i}^{\max }=4$. Also, we set the routing weights to $w_{i}^{k}=0$, and hence set $\gamma_{i}^{k, \max }=b_{i}^{\max }=4$.

First, we plot in Figure 2 the sample path of the total energy in the network at each time slot, illustrating the variability in the availability of energy due to energy harvesting process.

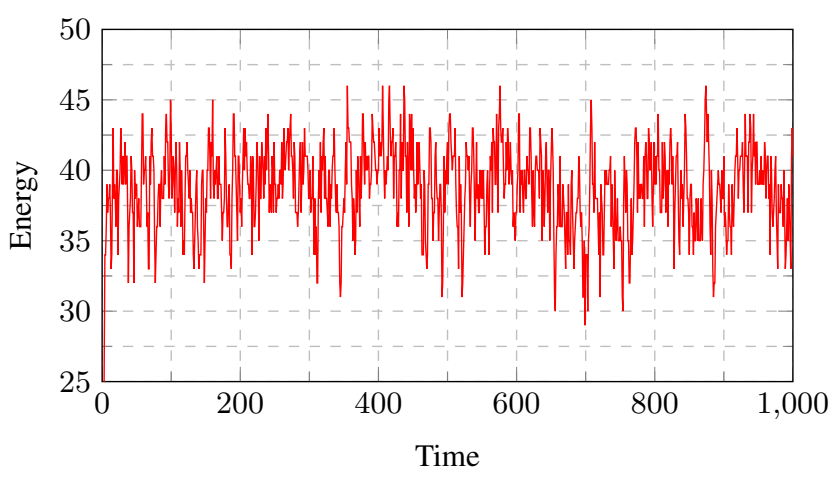

Fig. 2. Total energy in the network at each time slot.

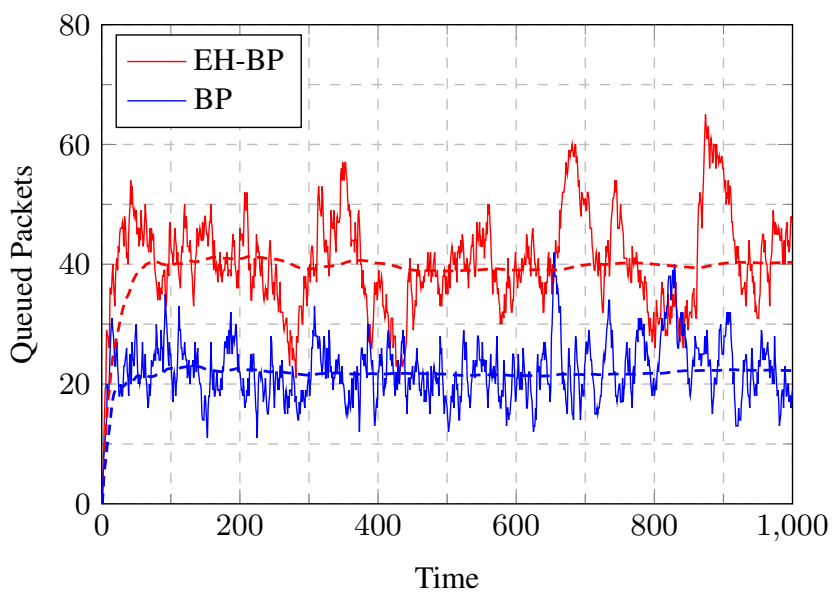

Fig. 3. Total amount of packets queued in the network at each time slot. Average values are shown in dashed lines.

In Figure 3 we plot a sample path of the total number of packets queued in the network at each time slot. For comparison we also show the backpressure algorithm (BP) when the nodes are powered by an infinite energy supply (equivalent to Algorithm 1 when setting $\left.\beta_{i}[t]=0\right)$. As expected both the EH-BP and BP policies stabilize the queues. Nonetheless, an increase in the variance of the queue dynamics as well as the average number of packets in the network can be observed for the EH-BP policy. This is due to the random nature of the energy harvesting process and as previously mentioned, using a positive weights $w_{i}^{k}$ can lead to lower average packets in the network at the expense of a larger battery capacity.

\section{CONCLUSIONS}

In this work, we have studied the problem of jointly routing and scheduling traffic in energy harvesting networks. We have proposed the energy harvesting backpressure (EH-BP) algorithm, which acts as a generalization of the backpressure policy to energy harvesting networks. Furthermore, we have provided theoretical guaranties on its network stabilization properties properties, which we have also validated by means of simulations. 


\section{REFERENCES}

[1] Ruud JM Vullers, RV Schaijk, Hubregt J Visser, Julien Penders, and Chris Van Hoof, "Energy harvesting for autonomous wireless sensor networks," IEEE Solid-State Circuits Magazine, vol. 2, no. 2, pp. 29-38, 2010.

[2] Jing Yang and Sennur Ulukus, "Optimal packet scheduling in an energy harvesting communication system," IEEE Transactions on Communications, vol. 60, no. 1, pp. 220-230, 2012.

[3] Kaya Tutuncuoglu and Aylin Yener, "Optimum transmission policies for battery limited energy harvesting nodes," IEEE Transactions on Wireless Communications, vol. 11, no. 3, pp. 1180-1189, 2012.

[4] Omur Ozel, Kaya Tutuncuoglu, Jing Yang, Sennur Ulukus, and Aylin Yener, "Transmission with energy harvesting nodes in fading wireless channels: Optimal policies," IEEE Journal on Selected Areas in Communications, vol. 29, no. 8, pp. 17321743, 2011.

[5] Chin Keong Ho and Rui Zhang, "Optimal energy allocation for wireless communications with energy harvesting constraints," IEEE Transactions on Signal Processing, vol. 60, no. 9, pp. 4808-4818, 2012.

[6] Miguel Calvo-Fullana, Javier Matamoros, and Carles AntonHaro, "Reconstruction of correlated sources with energy harvesting constraints," in European Wireless 2015; 21th European Wireless Conference; Proceedings of. VDE, 2015, pp. 16.

[7] O. Orhan, D. Gunduz, and E. Erkip, "Source-channel coding under energy, delay, and buffer constraints," IEEE Transactions on Wireless Communications, vol. 14, no. 7, pp. 38363849, July 2015.

[8] Paolo Castiglione, Osvaldo Simeone, Elza Erkip, and Thomas Zemen, "Energy management policies for energy-neutral source-channel coding," IEEE Transactions on Communications, vol. 60, no. 9, pp. 2668-2678, 2012.

[9] Miguel Calvo-Fullana, Javier Matamoros, and Carles AntónHaro, "Sensor selection and power allocation strategies for energy harvesting wireless sensor networks," IEEE Journal on Selected Areas in Communications, vol. 34, no. 12, pp. 36853695, 2016.

[10] Javier Matamoros, Miguel Calvo-Fullana, and Carles AntónHaro, "On the impact of correlated sampling processes in wsns with energy-neutral operation," in 2015 IEEE International Conference on Communications (ICC). IEEE, 2015, pp. 258263.

[11] Miguel Calvo-Fullana, Javier Matamoros, Carles Antón-Haro, and Sophie M. Fosson, "Sparsity-promoting sensor selection with energy harvesting constraints," in Acoustics, Speech and Signal Processing (ICASSP). 2016 IEEE International Conference on, March 2016.

[12] S. Ulukus, A. Yener, E. Erkip, O. Simeone, M. Zorzi, P. Grover, and K. Huang, "Energy harvesting wireless communications: A review of recent advances," IEEE Journal on Selected Areas in Communications, vol. PP, no. 99, pp. 1-1, 2015.

[13] Longbi Lin, Ness B Shroff, and R Srikant, “Asymptotically optimal energy-aware routing for multihop wireless networks with renewable energy sources," IEEE/ACM Transactions on networking, vol. 15, no. 5, pp. 1021-1034, 2007.
[14] Marios Gatzianas, Leonidas Georgiadis, and Leandros Tassiulas, "Control of wireless networks with rechargeable batteries," IEEE Transactions on Wireless Communications, vol. 9, no. 2, pp. 581-593, 2010.

[15] Longbo Huang and Michael J Neely, "Utility optimal scheduling in energy-harvesting networks," IEEE/ACM Transactions on Networking (TON), vol. 21, no. 4, pp. 1117-1130, 2013.

[16] Leandros Tassiulas and Anthony Ephremides, "Stability properties of constrained queueing systems and scheduling policies for maximum throughput in multihop radio networks," IEEE Transactions on Automatic Control, vol. 37, no. 12, pp. 19361948, 1992.

[17] Alejandro Ribeiro, "Ergodic stochastic optimization algorithms for wireless communication and networking," IEEE Transactions on Signal Processing, vol. 58, no. 12, pp. 63696386, 2010.

[18] Alejandro Ribeiro, "Stochastic soft backpressure algorithms for routing and scheduling in wireless ad-hoc networks," in Computational Advances in Multi-Sensor Adaptive Processing (CAMSAP), 2009 3rd IEEE International Workshop on. IEEE, 2009, pp. 137-140.

[19] Rick Durrett, Probability: theory and examples, Cambridge university press, 2010. 